Mathematical Research Letters 6, 439-447 (1999)

\title{
LATTICES IN KAC-MOODY GROUPS
}

\author{
Lisa Carbone and Howard Garland
}

\section{$\S 0$. Introduction}

Initially, we set out to construct non-uniform 'arithmetic' lattices in KacMoody groups of rank 2 over finite fields, as constructed by Tits [Ti1, Ti2] using the Bruhat-Tits tree of a Tits system for such groups. This attempt succeeded, and in fact, the construction we used can be applied to higher rank Kac-Moody groups over sufficiently large finite fields, and their buildings (Theorem 1.7 below). After completing this work, we learned that B. Remy has obtained an equivalent result for the more general class of almost split Kac Moody groups [R1, R2].

We have also constructed an uncountably infinite family of non-uniform lattices in the rank 2 Kac-Moody case, that is, we have succeeded in carrying over A. Lubotzky's construction of non-uniform lattices in $S l_{2}$ over a Laurent series field (Theorem 2.9 below). The basic tool for this extension is a (new) spherical Tits system (Theorems 2.2 and 2.7 below). It remains to determine whether, as in the case of $S l_{2}$, we have constructed uncountably many distinct conjugacy classes of non-uniform lattices within the Kac-Moody group.

In further analogy with Lubotzky's construction of lattices in $\mathrm{Sl}_{2}$, we have constructed an uncountably infinite family of cocompact lattice subgroups of rank 2 Kac-Moody groups. Once again, it remains to determine if there are uncountably many distinct conjugacy classes of these lattices.

In rank 2, the Kac-Moody groups and their lattice subgroups fail to have property $T$ (Proposition 4.1 below). In contrast to this, in the higher rank case, a result of Dymara and Januszkiewicz [DJ] implies that certain 'hyperbolic' KacMoody groups do have property $T$. Hence, the lattices that we construct in these cases are finitely generated and have finite commutator quotients.

Detailed proofs of the results mentioned above will appear elsewhere.

The authors would like to thank B. Remy and T. Januszkiewicz for their correspondence, and for informing us of their results. Thanks to A. Lubotzky for encouraging us to undertake this work and for explaining his constructions to us, and to H. Bass for many illuminating conversations.

Received June 11, 1999. 


\section{$\S 1$. The setting}

Let $A=\left(A_{i j}\right)_{i, j=1, \ldots, l}$ be an irreducible $l \times l$ symmetrizable generalized Cartan matrix. Let $\mathfrak{g}(A)$ be the Kac-Moody algebra over $\mathbb{C}$ corresponding to $A$. We have, as usual, the generators $e_{i}, f_{i}, h_{i}, i=1, \ldots, l$ of $\mathfrak{g}(A)(\mathrm{Kac},[\mathrm{K}])$. Let $\alpha_{i}$ be the simple root corresponding to $e_{i}, i=1, \ldots, l$. For a field $k$, let $G^{0}=G_{A}^{0}(k)$ be the minimal group associated to $A$ and $k$, as in Tits [Ti1], and let $G=G_{A}(k)$ be the corresponding completion, also constructed in [Ti1].

We have $B N$-pairs

$$
\begin{gathered}
\left(B^{0}, N\right) \text { in } G^{0}, \\
(B, N) \text { in } G,
\end{gathered}
$$

where $W \cong N / B^{0} \cap N \cong N / B \cap N$ is the Weyl group of the matrix $A$. In particular, we have Bruhat decompositions

$$
\begin{aligned}
G^{0} & =B^{0} W B^{0}, \\
G & =B W B .
\end{aligned}
$$

From now on, we assume that $k$ is a finite field. In this case, $G$ has the structure of a locally compact, totally disconnected, unimodular, topological group, with $B$ an open compact subgroup. The subgroups of $B$ of finite index constitute a basis of neighborhoods of the identity in $G$.

In $G^{0}$, there is an opposite (or twin) $B N$-pair $\left(B^{-}, N\right)$ (see [Ti2]). One has a second corresponding Bruhat decomposition

$$
G^{0}=B^{-} W B^{-},
$$

with $W \cong N / B^{-} \cap N$. We also have, in addition

$$
G^{0}=B^{-} W B^{0}
$$

From (1.6), we can easily deduce

$$
G=B^{-} W B,
$$

where we now regard $B^{-}$as a subgroup of the larger group $G$. We set $q=|k|$, the cardinality of the finite field $k$. We have the following:

Theorem 1.7. Let $P^{-} \subseteq G^{0}$ be a proper standard parabolic subgroup for the $B N$-pair $\left(B^{-}, N\right)$, (thus $\left.B^{-} \subseteq P^{-} \subsetneq G^{0}\right)$. Assume that the submatrix of $A$ corresponding to $P^{-}$is positive-definite. If $q>l$, then $P^{-}$is a non-uniform lattice in $G$.

The proof of Theorem 1.7 follows easily from (1.6a). One can compute the isotropy groups for $B^{-}$on the cosets $w \cdot B, w \in W$ in $G / B$, and obtain that suitably normalized, the covolume of $B^{-}$is given by the infinite series

$$
\sum_{w \in W} \frac{1}{q^{l(w)}},
$$

which is convergent (in fact, dominated by a convergent geometric series) provided $q>l$. 


\subsection{Remarks.}

(1) If $P^{-}$corresponds to a positive-definite submatrix of $A$, then $B^{-} \backslash P^{-}$is finite. Hence, if $B^{-}$is a lattice, then so is $P^{-}$.

(2) When $l=2$, one has that the Bruhat-Tits building $X$ associated to $(B, N)$ is a homogeneous tree of degree $q+1$. In this case, $B^{-}$is also a nonuniform lattice in the automorphism group of the $q+1$-homogeneous tree.

When $l=2$, the structure theory of discrete groups that act on trees with infinite quotient but finite covolume $[\mathrm{BL}]$ gives the following.

Corollary 1.9. When $l=2$, the group $B^{-}$is not finitely generated.

It follows that the group $B^{-}$cannot have Kazhdan's property $T$ (see $\S 4$ ). Corollary 1.9 is in contrast to the higher rank case where our lattices do have property $T$ and so are finitely generated (see $\S 5$ ).

We say that the matrix $A$ is hyperbolic if every proper subdiagram has corresponding matrix $A^{\prime}$ which is positive, semi-definite, but $A$ itself is neither classical nor euclidean.

This notion of hyperbolicity is closely related to, but somewhat different from the more usual notions such as those of [CS, Mo].

\section{$\S 2$. The tree case}

We now take $l=2$ and $A$ symmetric. We have

$$
A=\left(\begin{array}{cc}
2 & -m \\
-m & 2
\end{array}\right)
$$

for $m \geq 2$. When $m=2$, we say that $A$ is affine. For $m>2, A$ is hyperbolic. When $l=2$, the Weyl group $W$ is generated by two simple root reflections $w_{1}$, $w_{2}$, corresponding to the simple roots $\alpha_{1}, \alpha_{2}$. Let $X=X_{q+1}$ be the BruhatTits tree of the $B N$-pair $(B, N)$, and let $\mathcal{A}_{0,+}$ be the ray in $X$ whose edges are indexed by

$$
\{w \cdot B\}_{w \in W, l\left(w_{1} w\right)>l(w)},
$$

where $l($.$) is the length function on W$.

Let $\mathcal{B} \subseteq G$ be the stabilizer of the end determined by $\mathcal{A}_{0,+}$. Then

Theorem 2.2. $G=\mathcal{B} \sqcup \mathcal{B} w_{1} \mathcal{B}$.

Moreover, we can explicitly describe the structure of $\mathcal{B}$. To this end, we let $\Delta_{W}$ denote the set of Weyl roots of $\mathfrak{g}(A)$, and we set $t=w_{1} w_{2}$. Then we have:

$$
\Delta_{W}=\Delta_{W, 1} \sqcup \Delta_{W, 2},
$$

where

$$
\begin{aligned}
& \Delta_{W, 1}=\left\{-\alpha_{2},-w_{2} \alpha_{1},-w_{2} w_{1} \alpha_{2}, \ldots\right\} \cup\left\{\alpha_{1}, w_{1} \alpha_{2}, w_{1} w_{2} \alpha_{1}, \ldots\right\}, \\
& \Delta_{W, 2}=\left\{-\alpha_{1},-w_{1} \alpha_{2},-w_{1} w_{2} \alpha_{1}, \ldots\right\} \cup\left\{\alpha_{2}, w_{2} \alpha_{1}, w_{2} w_{1} \alpha_{2}, \ldots\right\},
\end{aligned}
$$


We let

$$
\mathcal{U}=\left\{\text { closed subgroup of } G \text { generated by all } \chi_{\alpha}(s)\right\},
$$

where $s \in k, \alpha \in \Delta_{W, 1}$, and

$$
\chi_{\alpha}(s)=\exp \left(s e_{\alpha}\right), e_{\alpha}=w e_{1}, \alpha=w \alpha_{1} .
$$

Let

$$
T=\left\{t^{n}\right\}_{n \in \mathbb{Z}},
$$

and

$$
\mathcal{B}_{I}=\bigcap_{w \in W} w B w^{-1}
$$

We have the following.

Theorem 2.7. $\mathcal{B}=\mathcal{U} T \mathcal{B}_{I}=\mathcal{U} \mathcal{B}_{I} T=\mathcal{B}_{I} T \mathcal{U} \ldots$

In particular,

$$
G / \mathcal{B}=\mathcal{B} / \mathcal{B} \sqcup\left(\mathcal{U} w_{1} \mathcal{B} / \mathcal{B}\right)=\left(w_{1} \mathcal{B} / \mathcal{B}\right) \sqcup\left(\mathcal{U}^{-} \mathcal{B} / \mathcal{B}\right),
$$

where $\mathcal{U}^{-}=w_{1}^{-1} \mathcal{U} w_{1}$.

The spherical building corresponding to Theorem 2.2 is 0 -dimensional, and may be identified with $G / \mathcal{B}$. By $(2.8)$, we have

$$
G / \mathcal{B} \cong(\mathcal{U} \sqcup \infty)
$$

which we may identify with $\partial X$, the boundary of the Bruhat-Tits tree $X=X_{q+1}$.

We let $\Delta$ be the subgroup of $G$ generated by all $\chi_{\alpha}(s)$, where $\alpha$ is negative, and $\alpha \in \Delta_{W, 1}$. Then $\Delta$ is a cocompact lattice in $\mathcal{U}$, and following Lubotzky ([L1]), we enlarge $\Delta$ to a non-uniform lattice in $G$ in the following way.

We let $g_{0}=i d, g_{1} \ldots, g_{q}$ in $P_{1}$ be a set of coset representatives for $P_{1} / B$, where $q=|k|$. For $i=0, \ldots, q$, let $\Delta_{i}=g_{i} \Delta g_{i}^{-1}$. In analogy with Lubotzky's construction of non-uniform lattices in $S l_{2}$ over a non-archimedean local field of characteristic $p>0$ [L1], and using Theorems 2.2 and 2.7, we have the following:

Theorem 2.9. Let $\Gamma$ be the group generated by $\Delta_{0}, \ldots, \Delta_{q}$. Then $\Gamma$ is a nonuniform lattice in $G$, and moreover, $\Gamma$ is the free product of the $\Delta_{i}, i=0, \ldots, q$.

The covolume of $\Gamma$, suitably normalized, is given by:

$$
1+(q+1) \sum_{i=1}^{\infty} \frac{1}{q^{i}}=\frac{2 q}{q-1},
$$

which is finite.

By varying the $g_{i}$ over the open set $B /\left(B \cap N_{G}(\Delta)\right)$, where $N_{G}(\Delta)$ denotes the normalizer in $G$ of $\Delta$, for $i=1, \ldots q$, we have an uncountably infinite family of non-uniform lattices here, parametrized by an open set of the form:

$$
\mathcal{P}(\Gamma, G)=B /\left(B \cap N_{G}(\Delta)\right) \times \cdots \times B /\left(B \cap N_{G}(\Delta)\right)
$$

( $q$ factors $)$. 
Lemma 2.11. We have $N_{G}(\Delta) \subseteq \mathcal{B}$.

The question arises as to whether we have uncountably many distinct conjugacy classes of lattices in $G$.

We have natural maps:

$$
\begin{aligned}
B /\left(B \cap N_{G}(\Delta)\right) \times & \cdots \times B /\left(B \cap N_{G}(\Delta)\right) \\
\downarrow & \downarrow \\
B /(B \cap \mathcal{B}) \times & \cdots \times B /(B \cap \mathcal{B}) \\
& \downarrow \\
G / \mathcal{B} \times & \cdots \times G / \mathcal{B}, \\
& \cong \\
(\mathcal{U} \sqcup \infty) \times & \cdots \times(\mathcal{U} \sqcup \infty) \\
& \cong \\
\partial X \times & \cdots \times \partial X .
\end{aligned}
$$

( $q$ factors).

We have the following:

Conjecture 2.12. The diagonal action of $G$ on

$$
G / \mathcal{B} \times \cdots \times G / \mathcal{B}
$$

(q factors), has the property that every orbit is nowhere dense.

An affirmative answer to Conjecture 2.12 would imply the existence of uncountably many conjugacy classes of non-uniform lattices in $G$.

Suppose that $A$ is affine, that is,

$$
A=\left(\begin{array}{cc}
2 & -2 \\
-2 & 2
\end{array}\right)
$$

Then $G$ is a central extension $\widehat{S l}_{2}$

$$
1 \longrightarrow k^{\times} \longrightarrow \widehat{S l}_{2} \longrightarrow S l_{2} \longrightarrow 1
$$

of $S l_{2}$ over a Laurent series field $\mathcal{L}_{k}$, in one variable, over the finite field $k$, by the (one dimensional) multiplicative group $k^{\times}$.

In this case, our uncountably infinite family of non-uniform lattices essentially coincides with Lubotzky's construction of uncountably many conjugacy classes of non-uniform lattices in $S l_{2}\left(\mathcal{L}_{k}\right)$ (see [L1] pp. 415), where Conjecture 2.12 
can be easily verified by dimension counting for $q \geq 4$, where $q=|k|$. Thus Conjecture 2.12 is true for $m=2$, and $q \geq 4$.

An affirmative answer to Conjecture 2.12 would also permit the following discussion about certain representation spaces of $\Gamma$ in $G$.

The non-uniform lattice $\Gamma$ that we constructed in Theorem 2.9 is not finitely generated (see also Corollary 1.9). Following A. Weil ([We]) we may obtain the following topological description of $\operatorname{Hom}(\Gamma, G)$. We may view $\operatorname{Hom}(\Gamma, G)$ as the subspace of all homomorphisms (with the induced topology) of the space

$$
G^{\Gamma}=\{f: \Gamma \longrightarrow G\}=\prod_{\gamma \in \Gamma} G
$$

of all maps from $\Gamma$ to $G$, with the product topology. Let

$$
\begin{aligned}
& \operatorname{Hom}_{0}(\Gamma, G)=\{\rho \in \operatorname{Hom}(\Gamma, G) \mid \rho \text { is injective, } \rho(\Gamma) \text { is discrete, } \\
&\text { has infinite fundamental domain and finite covolume. }\}
\end{aligned}
$$

We recall that our uncountably infinite family of non-uniform lattices is parametrized by an open set of the form (see 2.10):

$$
\mathcal{P}(\Gamma, G)=B /\left(B \cap N_{G}(\Delta)\right) \times \cdots \times B /\left(B \cap N_{G}(\Delta)\right)
$$

( $q$ factors). Any choice of the elements $\left(g_{1}, \ldots, g_{q}\right)$ (as in Theorem 2.9) from $\mathcal{P}(\Gamma, G)$ gives rise to a non-uniform lattice with fundamental domain isomorphic to that of $\Gamma$, and the same covolume as $\Gamma$.

We have a continuous map

$$
\phi: \mathcal{P}(\Gamma, G) \longrightarrow \operatorname{Hom}_{0}(\Gamma, G) .
$$

We ask the following:

Question 2.13. Is the image of $\mathcal{P}(\Gamma, G)$ open in $\operatorname{Hom}_{0}(\Gamma, G)$ ?

We do not even know if the answer to Question 2.13 is affirmative for Lubotzky's non-uniform lattices $\Gamma$ in $S l_{2}$ (see [L1] pp. 415 and [C]).

\section{$\S 3$. Cocompact lattices - the Schottky construction}

In the 'tree case'; that is, $l=2$, we can also exhibit an uncountably infinite family of cocompact lattices in $G$, once again, in analogy with Lubotzky's construction of cocompact lattices in a simple rank 1 group over a non-archimedean local field of characteristic $p \geq 0$. We use Lubotzky's generalization of the construction of classical Schottky groups of automorphisms of the upper-half plane. 
Theorem 3.1. When $l=2$, the group $G$ contains an uncountably infinite family of cocompact lattices which are finitely generated free groups that are generated by hyperbolic tree automorphisms (elements of infinite order which act as translations along a linear axis), and that act on the Bruhat-Tits tree $X=X_{q+1}$ with finite fundamental domain.

The uncountably infinite family of cocompact lattices is parametrized by an open set of the form:

$$
\mathcal{P}(\Gamma, G)=g_{1} B g_{1}^{\prime} \times \cdots \times g_{r} B g_{r}^{\prime},
$$

$\left(r=q^{2}\right.$ factors), where $g_{i}, g_{i}^{\prime} \in G$. Any choice $\left(g_{1} b_{1} g_{1}^{\prime}, \ldots, g_{r} b_{r} g_{r}^{\prime}\right) \in \mathcal{P}(\Gamma, G)$ generates a free cocompact lattice of rank $r=q^{2}$ in $G$, with the same fundamental domain as $\Gamma$.

We have the following:

Conjecture 3.3. The group $G$ contains uncountably many distinct conjugacy classes of cocompact lattices.

As in $\S 2$, Conjecture 3.3 is true when $A$ is affine, and we can verify the conjecture by dimension counting.

Since $\Gamma$ is free, we may identify $\operatorname{Hom}(\Gamma, G)$ with

$$
G \times \cdots \times G
$$

( $q^{2}$ factors, one for each generator of $\left.\Gamma\right)$. Let

$$
\operatorname{Hom}_{0}(\Gamma, G)=\{\rho \in \operatorname{Hom}(\Gamma, G) \mid \rho \text { is injective, }
$$

$\rho(\Gamma)$ is discrete, free and cocompact $\}$.

Let $\mathcal{P}(\Gamma, G)$ be as in 3.2 . We have a continuous map

$$
\phi: \mathcal{P}(\Gamma, G) \longrightarrow \operatorname{Hom}_{0}(\Gamma, G) .
$$

We have the following:

Conjecture 3.4. The image of $\mathcal{P}(\Gamma, G)$ is open in $\operatorname{Hom}_{0}(\Gamma, G)$.

We know that Conjecture 3.4 is true for Lubotzky's cocompact lattices in $\mathrm{Sl}_{2}$ (see [L1], pp. 407, and [C]).

\section{$\S 4$. Failure of property $T$ in rank 2}

If a property $T$ group $H$ acts on a tree, then the group $H$ must fix a vertex $([\mathrm{VH}])$. Therefore, if a non-compact group $G$ acts on a tree with compact vertex stabilizers, $G$ cannot have property $T$. This is the case for the group $G_{A}(k)$ in the case $l=2$, and thus we have the following. 
Proposition 4.1. When $l=2$, the group $G$ does not have Kazhdan's property $T$.

For a locally compact group $H$ containing lattices, $H$ has property $T$ if and only if lattices of $H$ have property $T$. Thus we obtain the following.

Corollary 4.2. When $l=2$, lattices of $G$ do not have Kazhdan's property $T$.

As we remarked in $\S 1$., Corollary 4.2 also follows from the fact that $B^{-}$cannot be finitely generated. Proposition 4.1 and Corollary 4.2 are in contrast to the higher rank case (see $\S 5$ ).

\section{$\S 5$. Cohomology}

In this section, we assume that all proper submatrices $A_{\theta}$ of $A$ of the form

$$
A_{\theta}=\left(A_{i j}\right)_{i, j \in \theta},
$$

where $\theta$ is a proper subset of $\{1, \ldots, l\}$, are positive definite. One can in fact weaken this assumption, but we make it here, nevertheless, for the sake of simplicity.

For $l \geq 3$, the methods of Garland in [G] yield results for various cohomologies on the Bruhat-Tits building $X$ associated with $(B, N)$, and on discrete subgroups $\Gamma \subseteq G$ (see also Ballmann-Swiatkowski [BSw], Pansu [P], and Zuk [Z]). For example, we have the following.

Theorem 5.1. Let $\Gamma \subseteq G$ be a cocompact lattice of $G$, and let

$$
\rho: \Gamma \longrightarrow \operatorname{Aut}(V)
$$

be a unitary representation $\Gamma$ in the complex Hilbert Space $V$ (not necessarily finite dimensional). If $l \geq 3$, and if $q=|k|$ is sufficiently large, then

$$
H^{i}(\Gamma, \rho)=0,0<i<l-1 .
$$

By Theorem 5.1 and $[\mathrm{VH}]$ we have the following.

Corollary 5.2. Given $G$ and $q$ as in the theorem, if $G$ contains a cocompact lattice, then $G$ has property $T$.

Applying the result of Valette and de la Harpe [VH] in this way has already been utilized in the works of Ballmann-Swiatkowski, Pansu and Zuk cited above.

We remark that we have not yet succeeded in constructing cocompact lattices in $G$ for $l \geq 3$.

During the preparation of this work, Dymara and Januszkiewicz brought their work to our attention [DJ]. They have also used the results in $[\mathrm{G}]$, to obtain vanishing theorems, but in addition, they applied the argument of the CasselmanWigner Theorem ([CW], Th. 2, pp. 209), to compute the continuous cohomology of $G$, and hence, in that way, to prove that $G$ has property $T$.

It then follows (see, in contrast, Corollary 4.2) that a lattice, (cocompact or non-uniform), $\Gamma \subseteq G$ has property $T$. In particular, $\Gamma$ is finitely generated and has finite commutator quotients. This is the case for the subgroups $P^{-}$as in Theorem 1.7. 


\section{References}

[BSw] W. Ballmann and J. Swiatkowski, On $L^{2}$-cohomology and property $(T)$ for automorphism groups of polyhedral cell complexes, Geom. Funct. Anal. 7 (1997), 615-645.

[BL] H. Bass and A. Lubotzky, Tree lattices, Birkhauser (1999), to appear.

[C] L. Carbone, Deformations of lattices in rank 1 groups of p-adic type, Proceeding of the International Conference on Computational and Combinatorial Algebra, Hong Kong (1999), Contemp. Math., in preparation.

[CS] D. Cartwright and M. Shapiro, Hyperbolic buildings, affine buildings and automatic groups, Michigan Math. J. 45 (1995), 511-523.

[CW] W. Casselman and D. Wigner, Continuous cohomology and a conjecture of Serre's, Invent. Math. 25 (1974), Springer-Verlag, 199-211.

[DJ] J. Dymara and T. Januszkiewicz, New Kazhdan groups (1999), preprint.

[G] H. Garland, p-adic curvature and the cohomology of discrete subgroups of p-adic groups, Ann. of Math. (2) 97 (1973), 375-423.

[K] V. Kac, Infinite dimensional lie algebras, Cambridge University Press (1990), Cambridge.

[L1] A. Lubotzky, Lattices in rank one lie groups over local fields, Geom. Funct. Anal. 1 (1991), 405-431.

[Mo] G. Moussong, Hyperbolic coxeter groups, PhD thesis, Ohio State University (1988).

[P] P. Pansu, Formule de Matsushima, de Garland, et propriete $(T)$ pour des groupes agissant sur des espaces symetriques ou des immeubles, Orsay (1996), preprint.

[R1] B. Remy, Formes presque deployees des groupes de Kac-Moody sur des corps quelconques (1999), preprint.

[R2] - Immeubles a courbure negative et theorie de Kac-Moody (1999), preprint.

[Ti1] Tits, J, Resume de cours - theorie des groupes, Annuaire du College de France (1980-1981), 75-87.

[Ti2] Uniqueness and presentation of Kac-Moody groups over fields, Journal of Algebra 105 (1987), 542-573.

[Ti3] _ Twin buildings and groups of Kac-Moody type, LMS Lecture Notes 165 (Proceedings of a conference on groups, combinatorics and geometry, Durham 1990), (1992), Cambridge University Press, 249-286.

[VH] A. Valette and P. de la Harpe, La propriete $(T)$ de Kazhdan pour les groupes localement compacts, Asterisque 175 (1989), Soc. Math. France.

[We] A. Weil, On discrete subgroups of lie groups, Ann. of Math. 72 (1960), 369-384.

[Z] A. Zuk, La propriete $(T)$ de Kazhdan pour les groupes agissant sur des polyedres, C. R. Acad. Sci. Paris Sér. I Math. 323 (1996), 453-458.

Department of Mathematics, Harvard University, One Oxford St., Cambridge, MA 02138

E-mail address: lisa@math.harvard.edu

Department of Mathematics, Yale University, 10 Hillhouse Avenue, P.O. Box 208283, New Haven, CT 06520

E-mail address: hgarland@math.yale.edu 